\title{
N-Acetylcysteine Resolves Placental Inflammatory-Vasculopathic Changes in Mice Consuming a High-Fat Diet
}

\author{
Lyda Williams, * Emmanuel S. Burgos, * Patricia M. Vuguin, ${ }^{\dagger}$ Clarence R. Manuel, ${ }^{\ddagger}$ Ryan Pekson, ${ }^{\S}$ Swapna Munnangi,
} Sandra E. Reznik, ${ }^{* * \dagger \dagger+\ddagger}$ and Maureen J. Charron ${ }^{*} \mathbb{\Phi}_{\ddagger \ddagger}$

From the Departments of Biochemistry, ${ }^{*}$ Cell Biology, ${ }^{\S}$ Medicine,${ }^{\natural}$ Pathology, ${ }^{\dagger \dagger}$ and Obstetrics and Gynecology and Women’s Health, ${ }^{\ddagger \ddagger}$ Albert Einstein College of Medicine, New York; the Departments of Pediatrics ${ }^{\dagger}$ and Pathology and Cell Biology, ${ }^{\ddagger}$ Columbia University Medical Center, New York; the Department of Surgery," Nassau University Health Center, East Meadow; and the Department of Pharmaceutical Sciences, ** St John's University, New York, New York

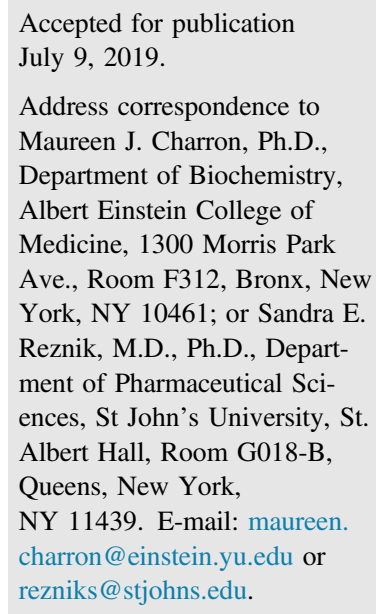

\begin{abstract}
The mechanism by which poor maternal nutrition can affect the long-term health of offspring is poorly understood. In mice, we previously found that maternal high-fat diet (HFD) exposure results in reduced fetal growth regardless of maternal genotype. We tested our hypothesis that maternal HFD-induced inflammation contributes to metabolic disease susceptibility of the offspring via alterations in the placenta. The effect of maternal genotype, diet, and treatment with the anti-inflammatory compound $\mathrm{N}$-acetylcysteine (NAC) on placental morphologic features was investigated. Placentas from wild-type dams maintained on a HFD but not those heterozygous (+/-) for Glut4 (Slc2a4) on the same diet had an increase in decidual inflammation and vasculopathy occurring together. NAC administration resulted in amelioration of HFD-induced decidual vasculopathy independent of offspring genotype and sex. Consistent with these morphologic improvements, placentas from HFD dams treated with NAC had decreased mRNA and immunostaining of IL-1 $\beta$ and monocyte chemoattractant protein-1, decreased mRNA of inflammatory genes, and increased mRNA of Vegfa. These results strongly suggest consumption of an HFD results in vascular changes in placenta reflected by alterations in expression of pivotal vascular developmental markers and inflammatory genes all of which are ameliorated by NAC. These placental changes play a key role in the increased programed metabolic disease of HFD-exposed offspring. (Am J Pathol 2019, 189: 2246-2257; https://doi.org/10.1016/j.ajpath.2019.07.010)
\end{abstract}

The number of deaths attributable to high body mass index and serum glucose has nearly doubled from 1980 to 2010 . $^{1}$ As of 2015 , almost $35 \%$ of all adults and $>50 \%$ of adults $>60$ years old in the United States were predicted to have metabolic syndrome, ${ }^{2}$ posing a tremendous burden on national health care costs. The developmental origin of health and disease hypothesis proposes an adverse intrauterine (IU) environment results in permanent alterations that increase susceptibility to metabolic disease and shortened life expectancy. ${ }^{3-8}$ We previously reported that the ability of the mother to use a high-fat diet (HFD) efficiently can differentially affect offspring metabolic disease susceptibility in a mouse model. ${ }^{9-11}$ Paradoxically, HFD Glut $4^{+/-}$ dams programed their male offspring for protection from metabolic disease, whereas the opposite was observed in male offspring from wild-type (WT) dams. Although all offspring were born intrauterine growth restricted (IUGR), only offspring from WT mothers had early-life catch-up growth. ${ }^{9}$ The effect of IUGR and catch-up growth can extend into adulthood and promote increased metabolic disease susceptibility. Although HFD exposure resulted in clear programing effects on offspring, the mechanism accounting for the adverse effect of HFD on the IU

Supported by NIH grants R21DK081194 (M.J.C.) and 1R01NS069577 (S.E.R.), American Diabetes Association grant 1-13-CE-06 (M.J.C.), a St. John's University seed grant (S.E.R.), the Nassau Health Care Corporation (S.E.R.), and an NIH Ruth Kirschstein predoctoral fellowship F31 DK093332 (L.W.).

S.E.R. and M.J.C. contributed equally to this work as senior authors. Disclosures: None declared. 
environment remained unclear. In the current study, we further interrogate the association between maternal genotype and the potentially adverse effects of HFD.

Maternal obesity is associated with increased inflammatory factors that influence placental function and result in adverse pregnancy outcomes via dysregulation of nutrient transport. $^{12} \mathrm{~N}$-acetylcysteine (NAC) has both antiinflammatory and antioxidant properties via direct amelioration of reactive oxygen species and by increasing intracellular reduced glutathione (GSH). ${ }^{13-15}$ Clinically, NAC has been used to ameliorate lipopolysaccharideinduced inflammatory cytokine response in maternal serum, placenta, and amniotic fluid. ${ }^{16}$ It has been safely used in pregnancy, where it increases the take-home infant rate in women with unexplained pregnancy loss. ${ }^{17}$ Finally, new data suggest that NAC may have neuroprotective and cardioprotective effects in the setting of IUGR. ${ }^{18}$ Using a well-established model of maternal HFD-induced inflammation and offspring susceptibility to metabolic disease, ${ }^{9-11}$ we determined the effect of maternal inflammation on placental structure and function and whether NAC treatment can prevent or alter these outcomes.

\section{Materials and Methods}

\section{Animals and Experimental Design}

All animal protocols were approved by the Albert Einstein College of Medicine Institutional Animal Care and Use Committee. In addition, all experiments that involved animals were conducted according to the NIH's Guide for the Care and Use of Laboratory Animals. ${ }^{19}$ As previously described, ${ }^{9-11}$ mice were housed in a barrier facility and maintained on a 14- to 10-hour light-dark cycle with ad libitum access to chow and water. Age- and body weight-matched 12- to 14-week-old female mice (CD1 background) were fed a control diet (Pico Lab Mouse Diet 5058; LabDiet, Fort Worth, TX) of 9\% fat, $20 \%$ protein, and $53 \%$ carbohydrate $(3.59 \mathrm{kcal} / \mathrm{g}$ ) or an HFD (product F3282; Bio-Serv, Flemington, NJ) of $35.5 \%$ fat, $20 \%$ protein, and $36.3 \%$ carbohydrate $(5.29 \mathrm{kcal} / \mathrm{g}) 2$ weeks before mating and throughout pregnancy. Both feeds supply calories derived from fat in the form of animal fat; the control diet substitutes some of the animal fat with soybean oil, whereas the HFD relies on animal fat alone. The use of these 2 diets side by side in studies aimed at investigating the effects of HFD on various aspects of mouse metabolic function has been well established in the literature. ${ }^{9-11}$

\section{Study 1}

Whether placental morphologic changes induced by HFD were dependent on maternal genotype was specifically investigated. ${ }^{9}$ WT females $(n=44)$ and Glut $4^{+/-}$females $(n=36)$ were bred to nonlittermate Glut $4^{+/-}$males (11 generations backcrossed onto CD1). Mice were maintained on either control or HFD and weighed daily. In summary, four experimental groups were included in study 1: wildtype control $\left(\mathrm{Ctrl}_{\mathrm{WT}}\right) \quad(n=24), \mathrm{Glut}^{+/-}$control $\left(\mathrm{Ctrl}_{\mathrm{Glut} 4+/-}\right)(n=18)$, wild-type HFD (HFD $\left.{ }_{\mathrm{WT}}\right)(n=20)$, and Glut $4^{+/-}$HFD $\left(\mathrm{HFD}_{\mathrm{Glut} 4+/-}\right)(n=18)$. Pregnancy was determined by the presence of a copulatory plug and defined as embryonic day (e) 0.5 . Pregnant dams were sacrificed by cervical dislocation at e18.5. Genotyping of offspring was performed as previously described. ${ }^{9-11}$ Fetuses were sacrificed by decapitation immediately after dissection from the uterine horn. A schematic of the study design is provided in Supplemental Figure S1A.

\section{Study 2}

WT females were bred to nonlittermate Glut $^{+/-}$males ( $n=72$ samples: 18 offspring per group, 3 litters per group, equal numbers of males and females per group, equal numbers of WT and Glut $4^{+/-}$offspring per group). Mice were maintained on either control or HFD and weighed daily. Genotyping of offspring was performed as previously described. ${ }^{9-11}$ At the presence of a copulatory plug, females were given vehicle (water) or NAC at a dose of $1 \mathrm{~g} / \mathrm{kg}$ in the drinking water from $\mathrm{e} 0.5$ until sacrifice. The amount of drinking water consumed was monitored, and NAC dosing was adjusted accordingly. In summary, four experimental groups were included in study 2: vehicle control $\left(\mathrm{Ctrl}_{\mathrm{Veh}}\right)$ $(n=18)$, NAC control $\left(\mathrm{Ctrl}_{\mathrm{NAC}}\right)(n=18)$, vehicle HFD $\left(\mathrm{HFD}_{\mathrm{Veh}}\right)(n=18)$, and NAC $\mathrm{HDF}\left(\operatorname{HFD}_{\mathrm{NAC}}\right)(n=18)$. Pregnant dams were sacrificed at e18.5, and fetuses were sacrificed as described above. Placental weight and fetal glucose was recorded as previously described. ${ }^{11,20} \mathrm{~A}$ schematic of the study design is provided in Supplemental Figure S1B.

\section{Placental Morphologic Findings}

Half of each placenta was flash-frozen in liquid nitrogen and stored at $-80^{\circ} \mathrm{C}$ after weighing; the other half was fixed in $10 \%$ neutral buffered formalin and embedded in paraffin. Sagittal full-face sections, $4 \mu \mathrm{m}$ in thickness, taken from the center of the placental disk and including the full thickness of the decidua and the full thickness of the labyrinth, were mounted and stained with hematoxylin and eosin.

\section{Microscopy and Scoring}

To evaluate placental vasculopathy and inflammation, hematoxylin and eosin-stained slides were scored by a practicing pathologist (S.E.R.), serving as the director of the perinatal pathology division at our center for the past 10 years, blinded to sample identity. The severity of inflammation and decidual vasculopathy was assessed by a grading system. For inflammation, scores of 0 to 3 were given for absent, mild, moderate, and marked inflammatory cell infiltrates in the maternal decidua, respectively. The three most active fields viewed at a magnification of $\times 400$ were identified, and the number of inflammatory cells 
present in each field was counted. For inflammation, a score of 0 was assigned if fewer than five inflammatory cells were present, a score of 1 was assigned for 5 to 10 inflammatory cells, a score of 2 for $>10$ but $<50$, and a score of 3 was assigned for fields containing $\geq 50$ inflammatory cells. For decidual vasculopathy, scores of 0 to 3 were given for absent, mild, moderate, and marked fibrinoid necrosis in the maternal decidual vasculature, respectively. Placentas that received positive scores for both inflammation and decidual vasculopathy were considered to have severe histopathologic changes. Images were photographed with a $10 \times$ objective lens on a Nikon digital sight camera attached to a Nikon Eclipse Ci light microscope. Total placenta area, junctional zone, and labyrinth zone area were determined with ImageJ version $1.49 \mathrm{v}$ (NIH, Bethesda, MD; https:// imagej.nih.gov/ij) as previously described. ${ }^{21}$

\section{Immunohistochemistry}

Sagittal full-face sections of formalin-fixed, paraffinembedded mouse placentas ( $n=6$ per group), $4 \mu \mathrm{m}$ thick, taken from the center of the placental disk and including the full thickness of the decidua and the full thickness of the labyrinth, were deparaffinized with xylene followed by rehydration using isopropyl alcohol, ethanol $(95 \%, 80 \%$, and $70 \%$ ), and distilled water. Heat-induced epitope retrieval was performed using sodium citrate buffer $(10$ $\mathrm{mmol} / \mathrm{L}$ sodium citrate, $0.05 \%$ Tween $20, \mathrm{pH} 6.0$ ) at $95^{\circ} \mathrm{C}$ to $98^{\circ} \mathrm{C}$. Endogenous peroxidase activity was neutralized using 3\% Hydrogen peroxide. Immunostaining was performed using Vectastain ABC Reagent Kit (Vector Laboratories, Burlingame, CA) following the manufacturer's protocol. Primary antibodies for IL-6 (Abcam, Cambridge, MA), monocyte chemotactic protein (MCP)-1 (Santa Cruz Biotechnology, Dallas, TX), and tumor necrosis factor (TNF)- $\alpha$ (Abcam) (1:200) were incubated overnight at $4^{\circ} \mathrm{C}$. Images were captured with a Nikon digital camera on a Nikon Eclipse Ci light microscope and imported to ImageJ version 1.48 for analysis. In addition, CD68, LY6, and terminal deoxynucleotidyl transferase-mediated dUTP nickend labeling immunohistochemical staining was performed on $5 \mu \mathrm{m}$ thick sections ( $n=3$ per group) by HistoWiz (Brooklyn, NY). Numbers of positively stained cells were quantified with QuPath version 0.1.2 (University of Edinburgh, Edinburgh, Scotland; https://qupath.github.io) analysis, performed by a blinded observer (S.M.).

\section{Quantitative Real-Time PCR Analysis}

Total RNA was extracted from placenta using TRIzol Reagent (Invitrogen, Carlsbad, CA), and first-strand cDNA was generated using SuperScriptIII (Invitrogen) according to the manufacturer's instructions. Quantitative real-time PCR was performed using the Roche 480 Real-Time PCR System with SYBR Green Master Mix (Roche, Indianapolis, IN). Primer sets were designed using Universal
Probe Library Assay Design Center. For quantitative analysis, all samples were normalized to cyclophilin B (Ppib), and relative mRNA expression levels were determined by the $\Delta \Delta$-CT method and expressed as fold change compared with $\mathrm{Ctrl}_{\mathrm{Veh}}$. Samples were measured in triplicate to assess technical variability ( $n=6$ to 8 per group from 4 to 6 litters). Primers are listed in Table 1.

\section{0xidative Stress Assays}

At $4{ }^{\circ} \mathrm{C}, 900 \mu \mathrm{L}$ of cold lysis buffer $(50 \mathrm{mmol} / \mathrm{L}$ potassium phosphate, $1 \mathrm{mmol} / \mathrm{L}$ EDTA, $1 \mathrm{mmol} / \mathrm{L}$ phenylmethylsulfonyl fluoride; $\mathrm{pH}$ 6.80) was added to each of 20 frozen half-placentas ( $n=5$ per group, representative of $n=3$ litters per group). Tissues were homogenized using a Tekmar Tissumizer Mark II. Samples were centrifuged $(15$ minutes, $16,000 \times g)$ and supernatants isolated. For catalase and oxidized glutathione (GSSG) reductase activities, aliquots were frozen and stored at $-80^{\circ} \mathrm{C}$. For GSH and GSSG analysis, deproteination of samples is required (metaphosphoric acid). All measures were performed in duplicate and assay results were normalized to protein concentration, whereas protein concentrations in samples tested for GSSG and GSH, GSSG reductase activity, and catalase activity were determined via Bradford method, protein concentrations in samples used for total antioxidant capacity (TAC), thiobarbituric acid reactive substances (TBARSs), and hydrogen peroxide assays were determined using bicinchoninic acid protein assay (Thermo Fisher, Waltham, MA).

\section{GSSG and GSH Analysis}

These metabolites were quantitated using a glutathione assay kit, following the manufacturer's instructions (Cayman Chemical, Ann Arbor MI). Before GSSG analysis, free GSH was derivatized with 2-vinylpyridine (SigmaAldrich, St. Louis, MO). Briefly, undiluted samples were quenched with $4 \mathrm{~mol} / \mathrm{L}$ triethanolamine solution. Reactions started on addition of enzyme reaction mix to samples. The increase of absorbance at $410 \mathrm{~nm}$ was recorded for $20 \mathrm{mi}-$ nutes using a SpectraMax 5 plate reader. For GSH quantitation, samples were further diluted three times and analyzed as above.

\section{GSSG Reductase Activity}

GSSG reductase activity was measured using a Cayman assay kit (Cayman Chemical), following the manufacturer's instructions. Briefly, after two dilutions, samples were added to assay mixtures. Enzymatic reactions started on addition of NADPH solution and monitored for 15 minutes at $340 \mathrm{~nm}$. Reaction rates were determined as described by manufacturer.

\section{Hydrogen Peroxide Assay}

Levels of hydrogen peroxide were measured using a Cell Biolabs (San Diego, CA) hydrogen peroxide assay kit, following the manufacturer's instructions. 
Table 1 Sequences of Primers Used for Quantitative RT-PCR

\begin{tabular}{|c|c|c|}
\hline Gene & Forward primer sequence & Reverse primer sequence \\
\hline Ppib & $5^{\prime}-$ CCGGGACAAGCCACTGAA-3' & 5'-GGCGAAGGGTTTCTCCACTT-3' \\
\hline Vegfa & $5^{\prime}$-GCAGCTTGAGTTAAACGAACG-3' & $5^{\prime}-\mathrm{GGTTCCCGAAACCCTGAG-3^{ \prime }}$ \\
\hline Pparg & $5^{\prime}$-GAAAGACAACGGACAAATCACC-3' & 5'-GGGGGTGATATGTTTGAACTTG-3' \\
\hline$I l 1 \beta$ & 5'-CTACAGGCTCCGAGATGAACAAC- $3^{\prime}$ & 5'-TCCATTGAGGTGGAGAGCTTTC-3' \\
\hline Mcp1 & $5^{\prime}$-GCCTGCTGTTCACAGTTGC-3' & $5^{\prime}-$ CAGGTGAGTGGGGCGTTA-3' \\
\hline$N f_{\kappa} b$ & 5'-CCAGCTTCCGTGTTTGTTCA-3' & 5'-AGGGTTTCGGTTCACTAGTTTCC-3' \\
\hline Tlr4 & 5'-GCACTCTGATCATGCCACTG-3' & $5^{\prime}$-CTGATCCATGCATGGTAGGT-3' \\
\hline Itgax & $5^{\prime}-$ GAGCCAGAACTTCCCAACTG-3' & 5'-TCAGGAACACGATGTCTTGG-3' \\
\hline Adrge1 & 5'-GGAGGACTTCTCCAAGCCTATT- 3' & 5'-AGGCCTCTCAGACTTCTGCTT-3' \\
\hline Slc2a1 & $5^{\prime}$-GGTGTGCAGCAGCCTGTGT-3' & $5^{\prime}-$ CACAGTGAAGGCCGTGTTGA-3' \\
\hline Mtor & 5'-TGATGTGCCGAGACCTTGAG-3' & 5'-GCTTGGATGTGATGACTTGCA-3' \\
\hline
\end{tabular}

\section{Catalase Activity}

The peroxidatic activity of catalase was determined using a Cayman assay kit (Cayman Chemical), following the manufacturer's instructions. Briefly, after 10 dilutions, samples were added to assay mixtures. Enzymatic reactions started on addition of hydrogen peroxide and continued for 20 minutes. The chromogen was added for 10 minutes before addition of potassium periodate. Formaldehyde formation was measured at $540 \mathrm{~nm}$.

\section{Lipid Peroxidation}

Lipid peroxidation was measured with a TBARS assay (Cayman Chemical), which measures malondialdehyde production, following the manufacturer's instructions.

\section{TAC}

TAC was determined by measuring copper reducing equivalents based on reduction of copper II to copper I, with a TAC assay kit from Cell Biolabs, following the manufacturer's instructions.

\section{Statistical Analysis}

Statistical analyses were performed using JMP software version 7.0 (SAS Institute, Cary, NC) or Graph Pad Prism software version 5.04 for Windows (Graph Pad Software, San Diego, CA). The severity of inflammation and decidual vasculopathy was assessed by a grading system. Data were segregated into ordinal variables in which the samples were assigned to a category showing an ordered progression in severity for inflammation and vasculopathy as 0 (normal), 1 (mild), 2 (moderate), and 3 (severe) (placentas with the higher scores were considered to have severe histopathologic changes) or nominal variables; in this case the scoring combined the presence of inflammation and vasculopathy and is presented as a binary variable based on the presence or absence of both inflammation and vasculopathy. To determine whether the placental inflammation and decidual vasculopathy (ordinal variable) were associated with fetal genotype, NAC treatment, placenta and litter weight, IU diet, maternal glucose at sacrifice, maternal weight, and the number of pups, a logistic regression analysis was used to test for associations. Differences in the proportion of placentas with the presence of both inflammation and vasculopathy (nominal variable) and maternal genotype, diet, and NAC treatment were examined. Pearson's $\chi^{2}$ test was the statistical test applied to sets of categorical data to evaluate how likely it is that any observed difference between the sets arose by chance. For continuous variables, data are presented as the means \pm SEM. Analysis of variance was used to test for significant differences between the means of two ( $t$-test) or more groups, and Bonferroni's posthoc analysis was performed. When appropriate, the two-tailed $U$-test was used. Acceptable study power was agreed $a$ priori to be $\geq 80 \%$ (type I error of $\leq 0.20$ ). $P<0.05$ was considered statistically significant. To confirm reproducibility of the data, all measurements were performed in duplicate or triplicate, as indicated above, and only representative results are shown in the figures.

\section{Results}

\section{$H_{F D_{\text {Veh }}}$ Pups Have Increased Blood Glucose}

Consistent with previous findings, ${ }^{9,22}$ no significant differences were found in total weight gain among the dams in the different experimental groups (Table 2). Similarly, no differences in placental area or area of junctional or labyrinth zones were observed (Figure 1). As expected, e18.5 HFD $\mathrm{Veh}_{\mathrm{V}}$ 
Table 2 Pup Characteristics at Embryonic Day 18.5

\begin{tabular}{|c|c|c|c|c|}
\hline Characteristic & Ctrlveh $_{\text {V }}$ & $\mathrm{Ctrl}_{\mathrm{NAC}}$ & $\mathrm{HFD}_{\text {Veh }}$ & $\mathrm{HFD}_{\mathrm{NAC}}$ \\
\hline Placenta weight, g & $0.131 \pm 0.005$ & $0.124 \pm 0.002$ & $0.124 \pm 0.003$ & $0.120 \pm 0.020$ \\
\hline Glucose, mg/dL & $49 \pm 2$ & $45 \pm 2$ & $69 \pm 5^{*}$ & $42 \pm 2$ \\
\hline
\end{tabular}

Data are expressed as means \pm SEM ( 4 to 6 litters per group with $n=40$ to 62 per group).

${ }^{\star} P<0.05$.

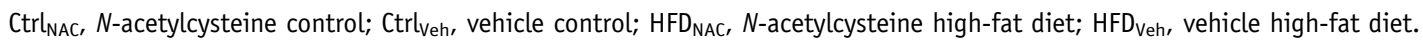

offspring had increased blood glucose levels that were normalized with the addition of NAC (Table 2).

\section{Maternal HFD Is Associated with Decidual Inflammation and Vasculopathy in WT Dams}

The amount of food consumed by the four groups in study 1 $\left(\mathrm{Ctrl}_{\mathrm{WT}}, \mathrm{Ctrl}_{\mathrm{Glut} 4+/-}, \mathrm{HFD}_{\mathrm{WT}}\right.$, and $\left.\mathrm{HFD}_{\mathrm{Glut} 4+/-}\right)$ was tracked by weighing the feed, and no significant differences were found among the four groups. When the placentas were analyzed by combining the presence or absence of both inflammation and vasculopathy (binary variable), the proportion of placentas with the presence of both inflammation and vasculopathy was significantly higher in WT (54\% and $65 \%$ ) when compared with Glut $4^{+/-}$dams $(11 \%$ and $16 \%)$ independent of the diet $(P<0.005)$ (Table 3$)$. When the placentas were analyzed based on the severity of inflammation plus the severity of the vasculopathy as 0 (normal), 1 (mild), 2 (moderate), and 3 (severe), the combination of severe inflammation and decidual vasculopathy (grade $\geq 3$ ) occurred most frequently in placentas from $\mathrm{HFD}_{\mathrm{WT}}$ dams $\left(55 \%\right.$ in $\mathrm{HFD}_{\mathrm{WT}}$ versus $33 \%$ in $\mathrm{Ctrl}_{\mathrm{WT}}$ versus $33 \%$ in $\mathrm{Ctrl}_{\mathrm{Glut} 4+/-}$ versus $38 \% \mathrm{HFD}_{\mathrm{Glut} 4+/-}$, $n=80, P=0.047$ ) (Table 4). The association of HFD and WT genotype with the most severe histopathologic changes (combination of inflammation and decidual vasculopathy), as determined by logistic regression analysis, was independent of maternal weight $(\beta=0.1, P=0.10)$, maternal serum glucose $(\beta=0.004, P=0.70)$, and or litter weight $(\beta=-0.13, P=0.20)$. Similarly, placental histologic changes were not associated with fetal genotype $(\beta=$ $-0.05, P=0.80)$, fetal sex $(\beta=0.35, P=0.12)$, placental weight $(\beta=8.8, P=0.40)$, litter weight $(\beta=0.35$, $P=0.2-)$, or the number of pups in litter $(\beta=-0.38$, $P=0.70)$.

Representative sections of decidua from each of the four experimental groups in study 1 are provided in Figures 2 and 3. The degree of decidual inflammation in placentas from the $\mathrm{HFD}_{\mathrm{WT}}$ dams (Figure 2B) appears to be much greater than in placentas from any of the other groups (Figure 2, A, C, and D). Arrows in Figure 2B indicate decidual macrophages, which were preferentially increased in placentas from $\mathrm{HFD}_{\mathrm{WT}}$ dams, as discussed below. A representative section of the increased decidual vasculopathy observed in $\mathrm{HFD}_{\mathrm{WT}}$ placentas as evidenced by fibrinoid necrosis is shown in Figure 3B; this histopathologic feature was absent in control placentas regardless of maternal genotype (Figure 3, A and C) and placentas from HFD $_{\text {Glut4+/- dams (Figure 3D). }}$

\section{Maternal HFD Does Not Increase Placental Labyrinthine Inflammatory Cell Infiltrates}

The placental labyrinth was investigated to determine whether morphologic changes observed in the decidua extended to this region. No inflammatory cell infiltrates were observed in the placental labyrinth regardless of maternal diet or genotype (Supplemental Figure S2).

\section{NAC Treatment Ameliorates HFD-Associated Decidual Vasculopathy}

The amount of food consumed by the four groups in study 2 $\left(\mathrm{Ctrl}_{\mathrm{Veh}}, \mathrm{Ctrl}_{\mathrm{NAC}}, \mathrm{HFD}_{\mathrm{Veh}}\right.$, and $\left.\mathrm{HFD}_{\mathrm{NAC}}\right)$ was tracked by weighing the feed, and no significant differences were found among the four groups. Representative placentas from $\mathrm{Ctrl}_{\text {Veh }}$ and $\mathrm{Ctrl}_{\mathrm{NAC}}$ showing no evidence of decidual vasculopathy are shown in Figure 4, A and B, whereas an example of severe vascular injury with fibrinoid necrosis is shown in Figure 4C. A striking amelioration of decidual vasculopathy was noted in placentas from $\mathrm{HFD}_{\mathrm{NAC}}$ dams (Figure 4D). This was accompanied by decreased Vegfa and increased Pgf mRNA levels in $\mathrm{HFD}_{\mathrm{NAC}}$ compared with $\mathrm{HFD}_{\text {Veh }}$ placenta (Figure 5). No significant difference in Ppar mRNA expression was measured among groups (Figure 5).

\section{NAC Treatment Partially Resolves HFD-Associated Decidual Inflammation}

Maternal HFD resulted in decidual inflammation (Figure 6), which was partially resolved by NAC supplementation (Figure 6). The proportion of placentas with the presence of both inflammation and decidual vasculopathy was significantly lower in placentas treated with NAC independently of the diet $(47 \%$ versus $25 \%$ in vehicle versus NAC, respectively, $n=72, P=0.0497$ ) (Table 5).

Immunohistochemical staining for macrophages (CD68positive cells) and neutrophils (LY6-positive cells) in the decidua of three randomly selected samples from each group in study 2 are shown in Supplemental Figure S3. No inflammatory infiltrates in the labyrinth were observed in response to HFD (Supplemental Figure S2). However, mRNA expression of the inflammatory cytokines $I l l b$ and Mcpl (Figure 5) was 
A

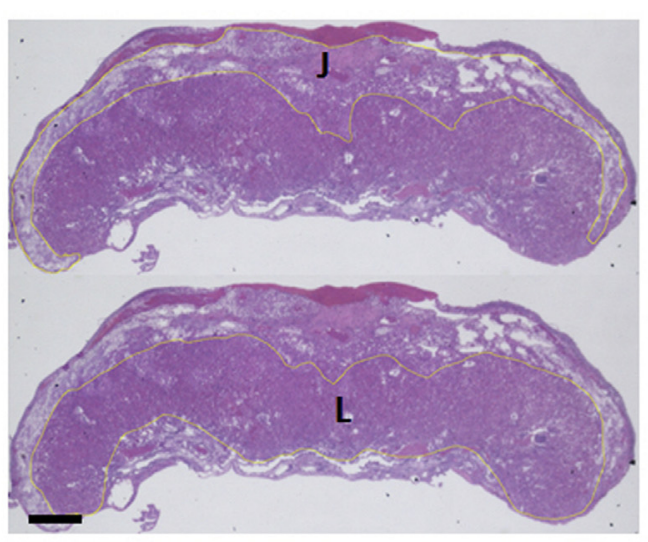

C

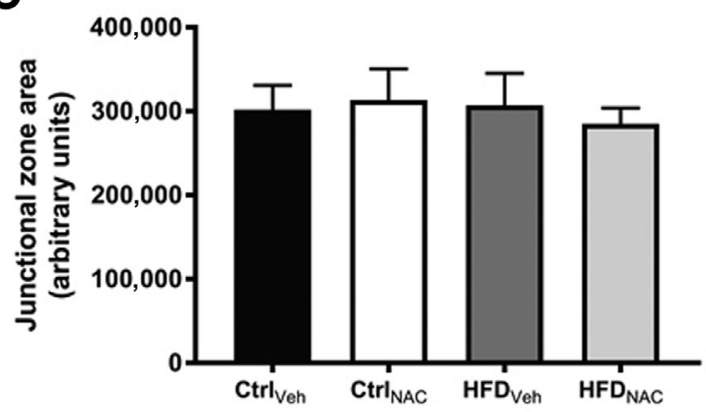

B

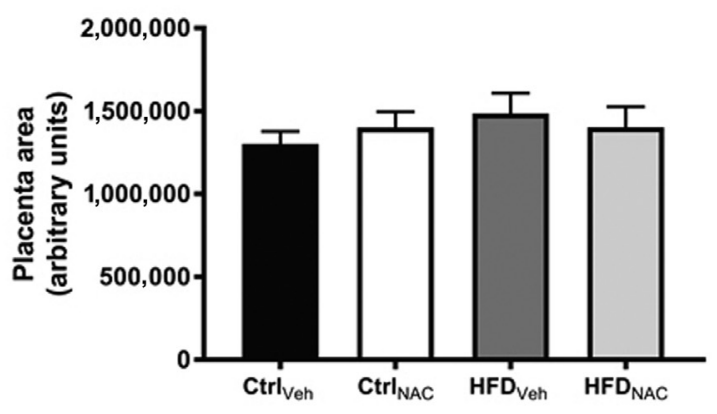

D

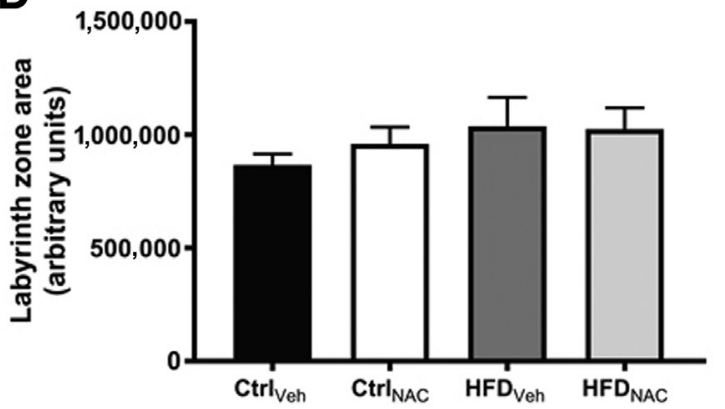

Figure 1 Area calculation of the placental zones. A: Representative images of outlined sections evaluated in calculating the area of the placental zones using the free-hand tool function in ImageJ version 1.48 (NIH, Bethesda, MD; https://imagej.nih.gov/ij). No significant differences were identified in the areas of the placenta (B), junctional zone (J) (C), or labyrinth zone (L) (D) regardless of maternal diet or treatment. $n=6$ per group. Scale bar $=500 \mu \mathrm{m}$. Original magnification, $\times 20$.

increased in HFD placentas and normalized by NAC treatment; an observation confirmed by immunohistochemical staining (Figures 7 and 8). The mRNA expression of the inflammatory genes Il6, Tnfa, NfKb, Tlr4, Itgax, and Adgrel in placenta was also decreased by NAC treatment of HFD-fed dams (Figure 5). No significant difference in immunostaining was observed for TNF- $\alpha$ (Supplemental Figure S4).

Table 3 Presence of Inflammation and Vasculopathy in WT and Glut4 $^{+/-}$Mice Exposed to Control or High-Fat Diet with or without $\mathrm{N}$-Acetylcysteine Treatment

\begin{tabular}{|c|c|c|}
\hline Group & $\begin{array}{l}\text { No inflammation and } \\
\text { or vasculopathy }\end{array}$ & $\begin{array}{l}\text { Inflammation and } \\
\text { vasculopathy }\end{array}$ \\
\hline $\operatorname{Ctrl}_{W T}(n=24)$ & $11(46)$ & $13^{*}(54)$ \\
\hline $\operatorname{HFD}_{W T}(n=20)$ & $7(35)$ & $13^{\dagger}(65)$ \\
\hline Ctrl $_{\text {Glut } 4+/-}(n=18)$ & $16(89)$ & $2(11)$ \\
\hline $\mathrm{HFD}_{\text {Glut4+/- }}(n=18)$ & $15(84)$ & $3(16)$ \\
\hline WT Ctrl Veh $_{\text {Veh }}(n=18)$ & $8(45)$ & $10^{\dagger}(55)$ \\
\hline WT $C \operatorname{trl} l_{\text {NAC }}(n=18)$ & $13(73)$ & $5(27)$ \\
\hline WT HFD Veh $_{\text {en }}(n=18)$ & $11(61)$ & $7(39)$ \\
\hline WT $\operatorname{HFD}_{\mathrm{NAC}}(n=18)$ & $14(78)$ & $4(22)$ \\
\hline
\end{tabular}

Data are expressed as $n(\%)$.

${ }^{*} P<0.01$.

${ }^{\dagger} P<0.05$.

Ctrl $_{\text {NAC, }}, N$-acetylcysteine control; Ctrl $_{\text {veh, }}$ vehicle control; HFD $_{\text {NAC }}$ $\mathrm{N}$-acetylcysteine high-fat diet; $\mathrm{HFD}_{\text {veh, }}$, vehicle high-fat diet; WT, wild type.
Paradoxically, $\mathrm{Ctrl}_{\mathrm{NAC}}$ resulted in increased expression of Illb mRNA compared with $\mathrm{Ctrl}_{\mathrm{Veh}}$ (Figure 5). Consistent with this, increased Tnf $\alpha$ and Itgax mRNA were also noted in $\mathrm{Ctrl}_{\text {NAC }}$ (Figure 5).

\section{NAC Treatment Increases Placental Glucose Transporter Expression in HFD Dams}

Expression of Slc2al and Slc2a3 mRNA was increased in $\mathrm{HFD}_{\mathrm{NAC}}$ compared with $\mathrm{HFD}_{\mathrm{Veh}}$ but not in $\mathrm{Ctrl}_{\mathrm{Veh}}$ compared with $\mathrm{Ctrl}_{\mathrm{NAC}}$ placenta (Figure 5). In contrast, no

Table 4 Presence of Mild, Moderate, and or Severe Inflammation Plus Vasculopathy in Wild-Type and Glut4 ${ }^{+/-}$Deciduas Exposed to Control or High-Fat Diet

\begin{tabular}{lcllr}
\hline $\begin{array}{l}\text { Inflammation plus } \\
\text { vasculopathy }\end{array}$ & None & Mild & Moderate & \multicolumn{1}{c}{ Severe } \\
\hline Ctrl $_{\text {WT }}(n=24)$ & $3(12)$ & $7(30)$ & $6(25)$ & $8(33)$ \\
HFD $_{\text {WT }}(n=20)$ & $1(5)$ & $5(25)$ & $3(15)$ & $11^{*}(55)$ \\
Ctrl $_{\text {Glut4+/- }(n=18)}$ & $9(50)$ & $3(17)$ & $0(0)$ & $6(33)$ \\
HFD $_{\text {Glut4+/- }(n=18)}$ & $10(56)$ & $1(6)$ & $0(0)$ & $7(38)$ \\
\hline
\end{tabular}

Data are expressed as $n(\%)$.

${ }^{*} p<0.05$.

$\mathrm{Ctrl}_{\mathrm{wt}}$, wild-type control; $\mathrm{Ctrl}_{\mathrm{Glut} 4+/-,} \mathrm{Glut4}^{+/-}$control; $\mathrm{HFD}_{\mathrm{Glut}_{4+/-} \text {, }}$ Glut4+/- high-fat diet; $\mathrm{HFD}_{\mathrm{WT}}$, high-fat diet wild type. 


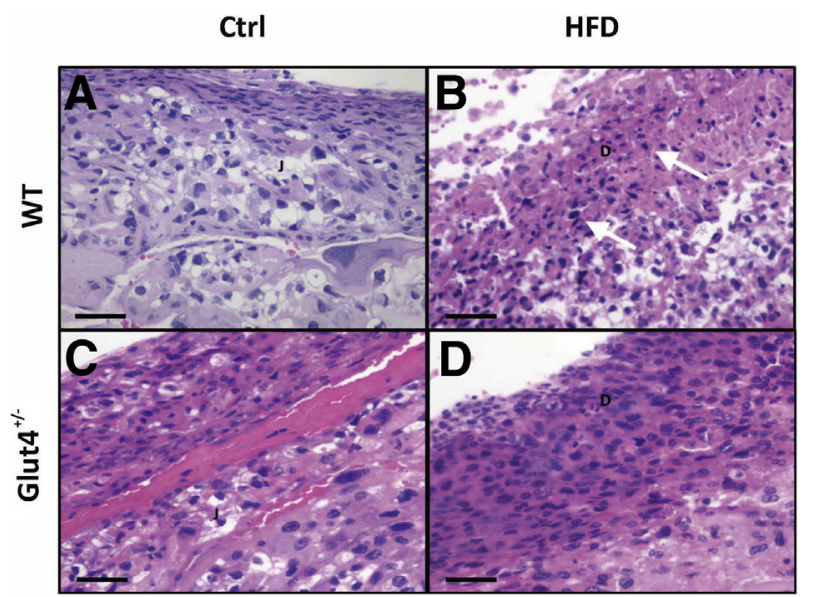

Figure 2 Placentas from Glut4 ${ }^{+/-}$dams are protected from high-fat diet (HFD)-induced inflammation. A, C, and $\mathbf{D}$ : No evidence of inflammation in wild-type (WT) control (Ctrl WT) (A), $\mathrm{Glut}^{+/-}$control (Ctrl ${ }_{\text {Glut4+/- }}$ ) (C), or Glut4 ${ }^{+/}$HFD $\left(\mathrm{HFD}_{\mathrm{Glut} 4+/-}\right)$ (D) placentas. B: Increased decidual inflammation in WT HFD ( HFD $\left._{\mathrm{WT}}\right)$ placentas. Arrows indicate prominent inflammatory cells. $n=6$ per group. Scale bars $=50 \mu \mathrm{m}$. Original magnification, $\times 400$. D, decidua; $\mathrm{J}$, junctional zone.

difference in expression of the amino acid transporters Slc38al, Slc38a2, and Slc38a4 or Mtor mRNAs was measured in response to maternal diet or NAC treatment (Figure 5).

\section{Maternal HFD Is Associated with Increased Placental 0xidative Stress, Which Is Paradoxically Accentuated by NAC}

The effect of maternal HFD on several measures of oxidative stress in the placenta is shown in Figure 9. Although the changes were not significant when compared with levels in $\mathrm{Ctrl}_{\text {Veh }}$ dams, HFD increased levels of GSSG (Figure 9A) and

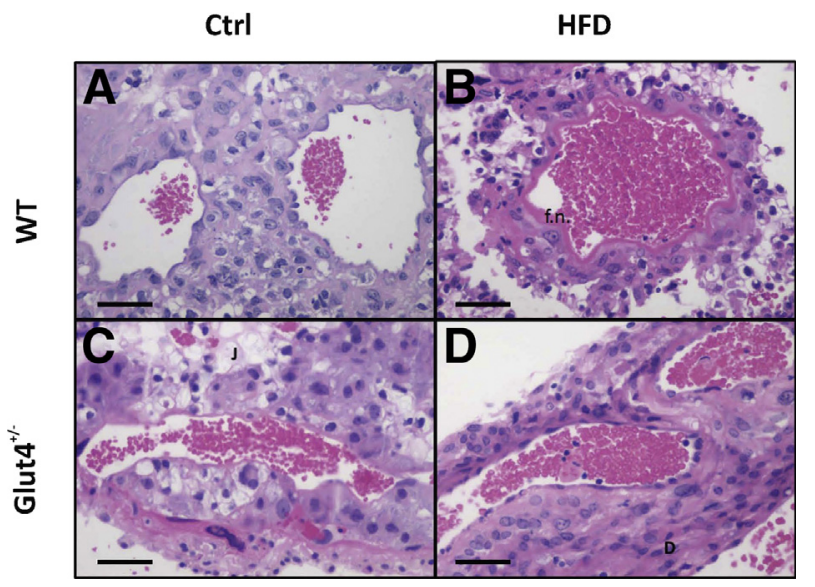

Figure 3 Placentas from Glut4 ${ }^{+/-}$dams are protected from high-fat diet (HFD) -induced decidual vasculopathy. A, C, and D: Normal vessels in the decidua in wild-type (WT) control (Ctrl WT $_{\text {T }}$ (A), Glut4 $^{+/-}$control (Ctrl Glut $4+/-_{\text {- }}$ ) (C), and Glut4 ${ }^{+/-}$HFD $\left(\mathrm{HFD}_{\mathrm{Glut}_{4+-}}\right)$ (D) placentas. B: Decidual vasculopathy in WT HDF ( $\mathrm{HFD}_{\mathrm{WT}}$ ) placentas as evidenced by fibrinoid necrosis (f.n.). $n=6$ per group. Scale bars $=50 \mu \mathrm{m}$. Original magnification, $\times 400$. D, decidua; $\mathrm{J}$, junctional zone.

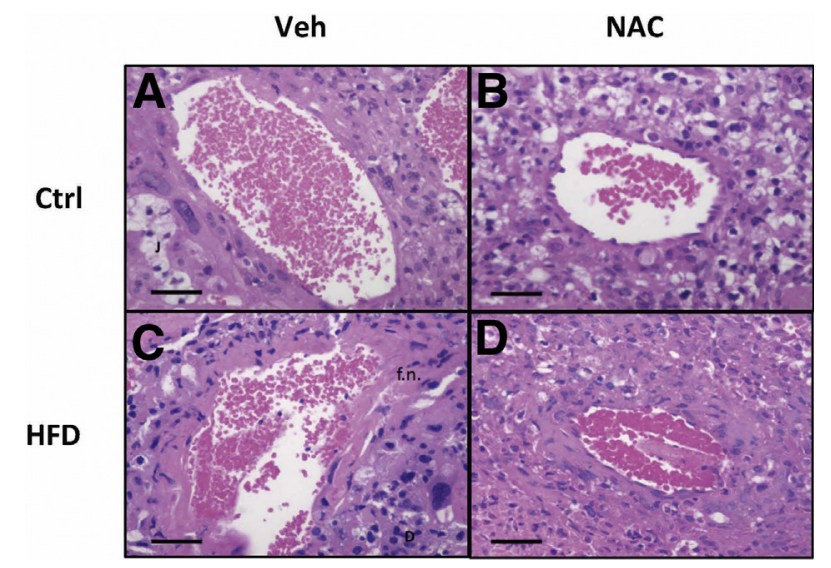

Figure $4 \mathrm{~N}$-acetylcysteine (NAC) treatment ameliorates decidual vasculopathy in placenta from wild-type (WT) dams on a high-fat diet (HFD). A and $\mathbf{B}$ : Normal vessels with no evidence of fibrinoid necrosis in vehicle control (Ctrl $\left.V_{\text {eh }}\right)$ (A) and NAC control (Ctrl $\mathrm{CAC}_{\text {NC }}$ (B) placentas. C and D: Fibrinoid necrosis (f.n.) of vessels in vehicle HFD (HFD veh) placentas, which is ameliorated with NAC treatment (D). $n=18$ per group. Scale bars $=50$ $\mu \mathrm{m}$. Original magnification, $\times 400 . \mathrm{D}$, decidua; J, junctional zone.

TBARS (Figure 9F) and reduced GSH/GSSG ratio (Figure 9B) and TAC (Figure 9G) in placental lysates. GSSG reductase and catalase levels were unaffected by diet (Figure 9, C and E, respectively). Although hydrogen peroxide levels were decreased by NAC in placentas from control dams, HFD consumption alone had no significant effect on hydrogen peroxide (Figure 9D). On the basis of terminal deoxynucleotidyl transferase-mediated dUTP nick-end labeling staining, there were no differences in the numbers of apoptotic cells among the four groups (data not shown).

Interestingly, NAC did not attenuate the prooxidant effects produced by maternal HFD and, in fact, accentuated these effects. Specifically, NAC appeared to potentiate the slight increase in GSSG and decrease in GSH/GSSG ratio produced by HFD (Figure 9, A and B), although these effects were not statistically significant. Although HFD alone $\left(\mathrm{HFD}_{\mathrm{Veh}}\right)$ had no significant effect on placental hydrogen peroxide levels or catalase activity, the combination of HFD and NAC ( $\left.\mathrm{HFD}_{\mathrm{NAC}}\right)$ slightly increased both hydrogen peroxide concentration and catalase activity compared with $\mathrm{HFD}_{\text {Veh }}$ (Figure 9, D and E). Although the increase in TBARS produced by maternal consumption of HFD was not significant when compared with $\mathrm{Ctrl}_{\mathrm{Veh}}$, the combination of maternal HFD and NAC produced a significant increase in lipid peroxidation $(P<0.01)$ (Figure 9F). Similarly, the decrease in TAC associated with maternal HFD was accentuated on NAC treatment $(P<0.01)$ (Figure 9G), although this finding was not significant when compared with HFD alone.

\section{Discussion}

Using an established animal model of HFD-induced fetal programing of adult disease, ${ }^{9-11}$ we found that the adverse effects observed in offspring born to these dams stem from 
A

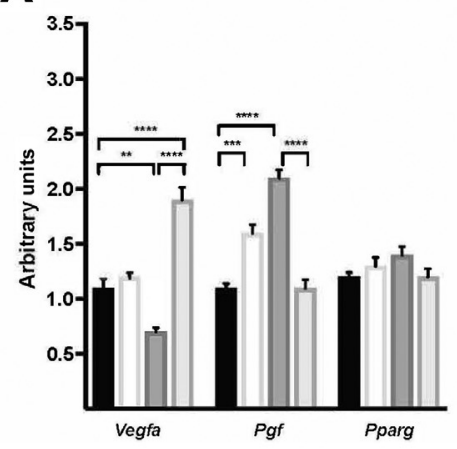

B

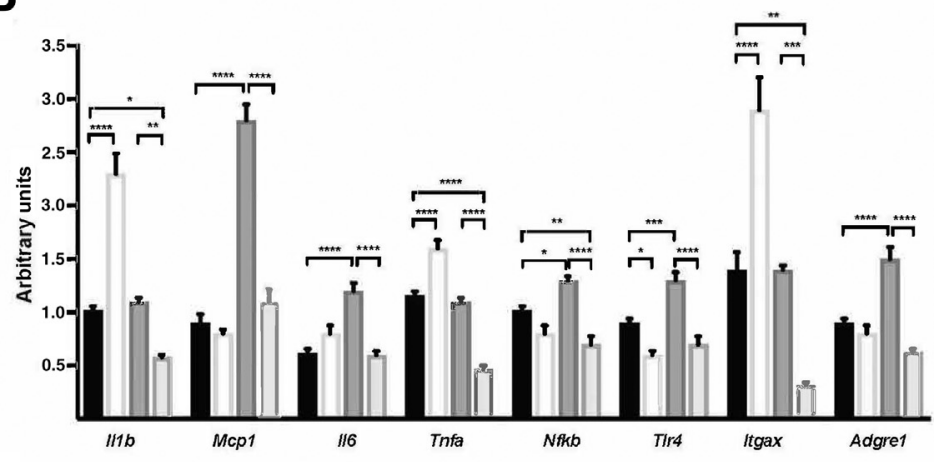

C

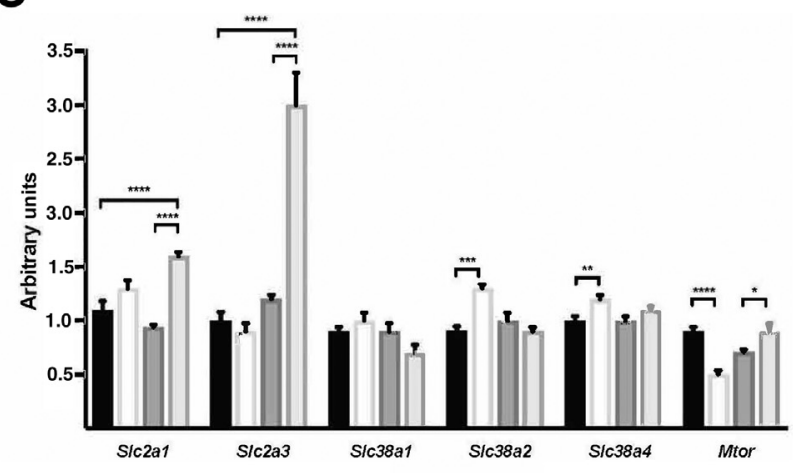

Figure 5 High-fat diet (HFD) and $\mathrm{N}$-acetylcysteine (NAC) treatment affect placental responses by altering gene expression. Relative expression of the indicated genes in vehicle control ( $\left(\mathrm{Ctrl}_{\mathrm{Veh}}\right), \mathrm{NAC}$ control $\left(\mathrm{Ctrl}_{\mathrm{NAC}}\right)$, vehicle HFD $\left(\mathrm{HFD}_{\text {veh }}\right)$, and NAC HFD (HFD $\left.{ }_{\mathrm{NAC}}\right)$ mice is plotted. Genes involved in vascular development $(\mathbf{A})$, oxidative stress and inflammation (B), and nutrient transport (C) are shown. ${ }^{*} P<0.05,{ }^{* *} P<0.01,{ }^{* * *} P<0.001$, and $* * * * P<0.0001$. placental morphologic alterations and expression of cytokine and developmental markers. Our findings indicate that exposure to maternal HFD results in alterations in placental vasculature with increases in decidual fibrinoid necrosis and inflammation. However, alterations were observed only in placentas from $\mathrm{HFD}_{\mathrm{WT}}$ dams, whereas placentas from $\mathrm{HFD}_{\mathrm{Glut} 4+/-}$ dams were unaffected. It was previously

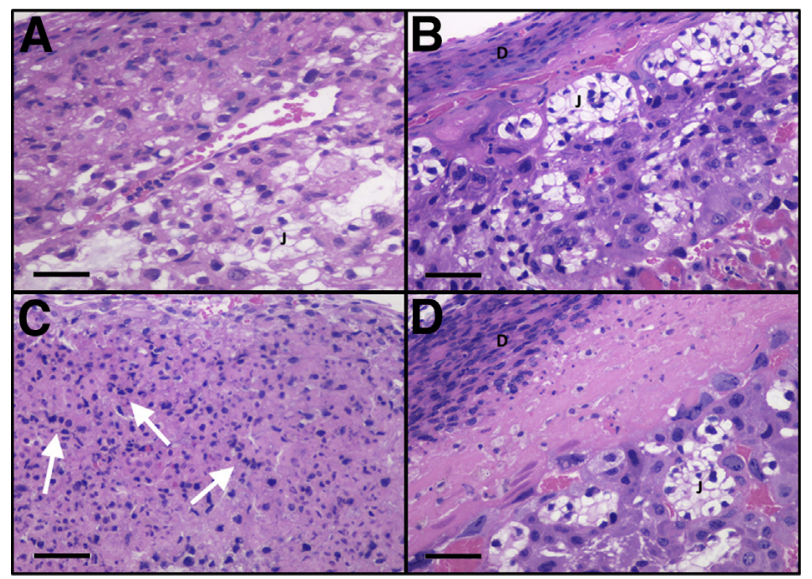

Figure $6 \quad \mathrm{~N}$-acetylcysteine (NAC) treatment partially resolves high-fat diet (HFD)-induced inflammation of the decidua. No evidence of inflammation in decidua of vehicle control (Ctrlveh) (A) or NAC control ( $\mathrm{Ctrl}_{\mathrm{NAC}}$ ) (B) placentas. Significant inflammation in decidua of vehicle HFD ( $\left.\mathrm{HFD}_{\text {veh }}\right)$ dams (clusters of inflammatory cells indicated by arrows) (C), which is partially resolved with NAC treatment (D). $n=18$ per group. Scale bars $=50 \mu \mathrm{m}$. Original magnification, $\times 400 . \mathrm{D}$, decidua; $\mathrm{J}$, junctional zone. reported that the protection from metabolic disease in these offspring resulted from the effective maternal use of dietary fat as a substrate by Glut $4^{+/-}$dams. ${ }^{9}$ A similar mechanism might be protective here, although additional studies beyond the scope of this report would be needed to identify the changes in molecular pathways in the Glut $4^{+/-}$dams that lead to protection. Although it was previously reported that the offspring exposed to a maternal HFD were adversely affected, the role of the uteroplacental vasculature in this setting was unknown. ${ }^{9-11}$ Furthermore, we investigated, for the first time, whether HFD-induced placental morphologic changes could be resolved by NAC administration to WT dams.

Changes in histomorphologic features, vasculogenesis, angiogenesis, and nutrient transfer capacity can have effects on the offspring. Our findings indicate that maternal HFD consumption leads to increased placental production of inflammatory cytokines, which in turn may affect production

Table 5 Presence of Inflammation and Vasculopathy in WildType Mice Exposed to Vehicle or NAC Treatment

\begin{tabular}{lll}
\hline Exposure & $\begin{array}{l}\text { No inflammation } \\
\text { and or vasculopathy }\end{array}$ & $\begin{array}{l}\text { Inflammation and } \\
\text { vasculopathy }\end{array}$ \\
\hline Water & $19(53)$ & $17^{\star}(47)$ \\
NAC Treatment & $27(75)$ & $9(25)$ \\
\hline
\end{tabular}

Data are expressed as $n(\%)$.

${ }^{*} P<0.05$.

NAC, $\mathrm{N}$-acetylcysteine. 


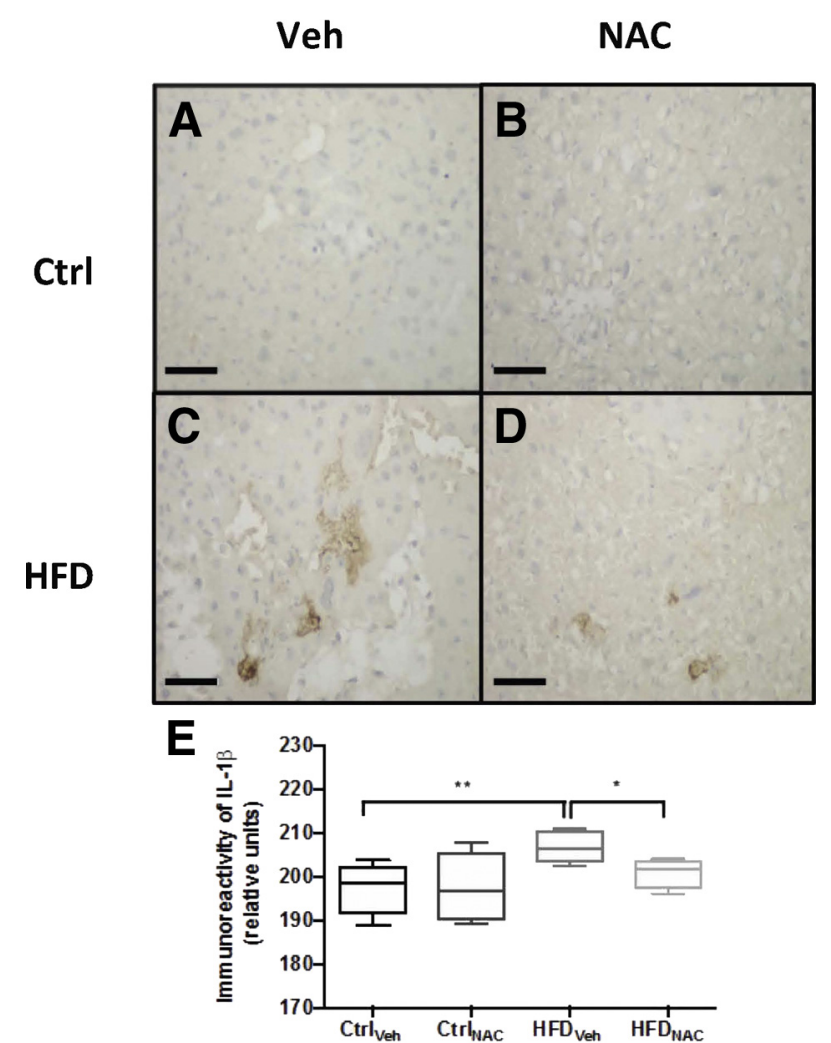

Figure 7 Increased IL-1 $\beta$ immunoreactivity in labyrinth of vehicle high-fat diet (HFD) ( $\mathrm{HFD}_{\text {veh }}$ ) placenta is ameliorated with $\mathrm{N}$-acetylcysteine (NAC) treatment. A and B: Minimal immunohistochemical staining of IL-1 $\beta$ in labyrinth of vehicle control ( $\left.\mathrm{Ctrl}_{\text {veh }}\right)$ (A) and NAC control ( $\left.\mathrm{Ctrl}_{\mathrm{NAC}}\right)$ (B) placentas. $\mathbf{C}$ and $\mathbf{D}$ : Increased IL-1 $\beta$ in $\mathrm{HFD}_{\text {veh }}$ labyrinth $(\mathbf{C})$ is reduced to control levels by NAC treatment (D). E: Box and whisker plot of IL-1 $\beta$ immunoreactivity. $n=6$ per group. Scale bars $=50 \mu \mathrm{m}$. Original magnification, $\times 400 .{ }^{*} P<0.05,{ }^{* *} P<0.01$.

of angiogenic factors and vessel morphologic features. Decidual vasculopathy reduces blood flow to the placenta; thus, the fetuses, ironically, in the setting of maternal overnutrition, are undernourished. In line with our findings, in nonhuman primate models, maternal HFD resulted in reduced fetal weight ${ }^{23}$; reduction in uterine artery blood flow $^{24}$; increased mRNA expression of $I L 1 B, M C P 1$, and $T L R r 4^{24}$; changes in the fetal hypothalamic pituitary axis, including increased expression of $I L I B$ and $I L I$ type 1 receptor $^{25}$; and fetal behavioral changes. ${ }^{26}$ Although the published studies have discordant outcomes in response to IU exposure to maternal HFD diet, likely because of differences in animal models, composition of the HFD diet, and/or timing of the insult, these studies unequivocally found that maternal HFD affects fetal growth, blood flow, and nutrient supply to the fetus, all of which can program changes in the physical and neurodevelopment of offspring. $^{27,28}$

Although there were no significant differences in placental weights, the effect of altered vasculature on nutrient transport was investigated by determining mRNA expression of glucose and amino acid transporters as well as
Mtor, which acts as a placental nutrient sensor. ${ }^{12,29}$ Interestingly, although the glucose levels of $\mathrm{HFD}_{\mathrm{veh}}$ pups were significantly increased, the expression of Slc2al and Slc2a3 mRNA was decreased in comparison to $\mathrm{HFD}_{\mathrm{NAC}}$. Although decreased mammalian target of rapamycin activity in placenta has been associated with IUGR, ${ }^{29}$ no significant difference in the expression of amino acid transporters or Mtor was detected.

Although maternal body size and placental size can predict later development of coronary disease in human cohorts $^{30,31}$ and maternal HFD in a rat model and caloric restriction in a mouse model resulted in reduced junctional zone area but preservation of the labyrinth zone, ${ }^{32,33}$ no significant differences were observed in placental area, junctional zone area, or labyrinth zone area in our model. The reasons underlying the differences between our model and others may be related to strain differences, as well as differences in the composition and/or timing of the nutritional intervention. Future studies will be needed to tease apart the contributions of these factors.

In our hands, maternal HFD in WT dams increases the frequency of inflammation and decidual vasculopathy

\section{Veh NAC}

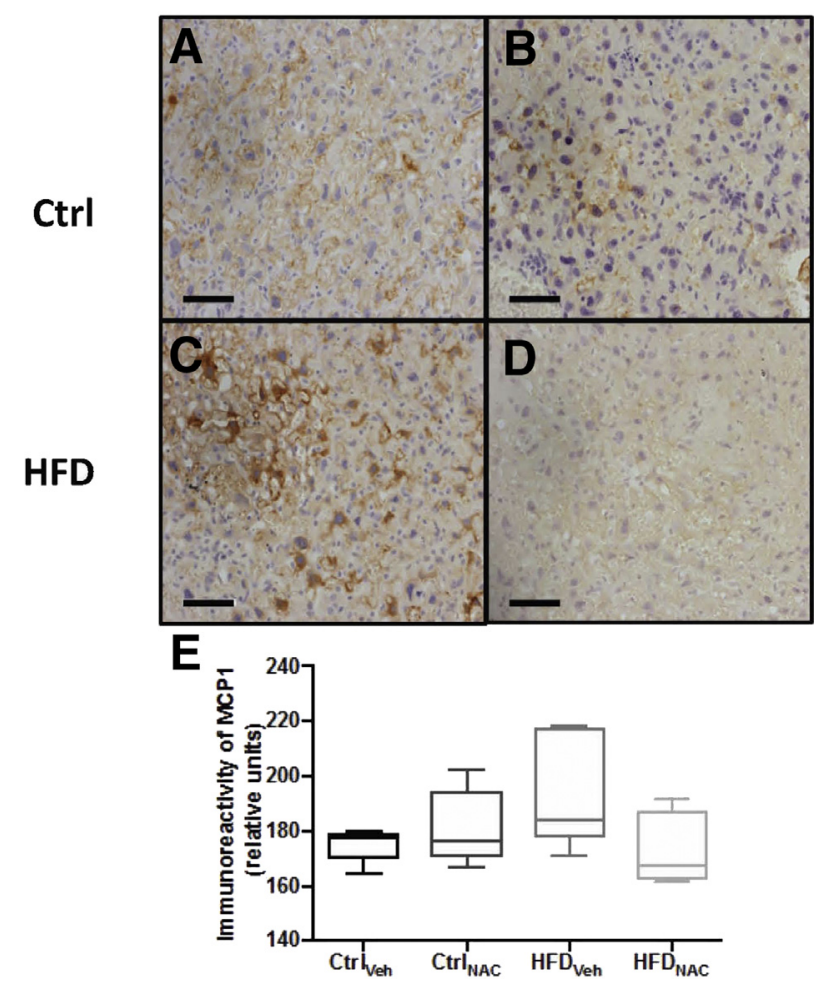

Figure 8 Variable monocyte chemoattractant protein (MCP)-1 in labyrinth of vehicle high-fat diet ( $\left.\mathrm{HFD}_{\mathrm{veh}}\right)$ placenta. A and B: No significant difference in MCP1 expression in labyrinth of vehicle control $\left(\mathrm{Ctrl}_{\text {veh }}\right)\left(\right.$ A) or $\mathrm{N}$-acetylcysteine (NAC) control ( Ctrl $\left._{\text {NAC }}\right)$ (B) placentas. C and $\mathbf{D}$ : Variable MCP1 expression in vehicle HFD ( HFD $_{\text {veh }}$ ) placentas (C)

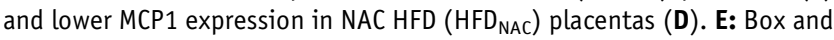
whisker plot of MCP1 immunoreactivity. $n=6$ per group. Scale bars $=50$ $\mu \mathrm{m}$. Original magnification, $\times 400$. 

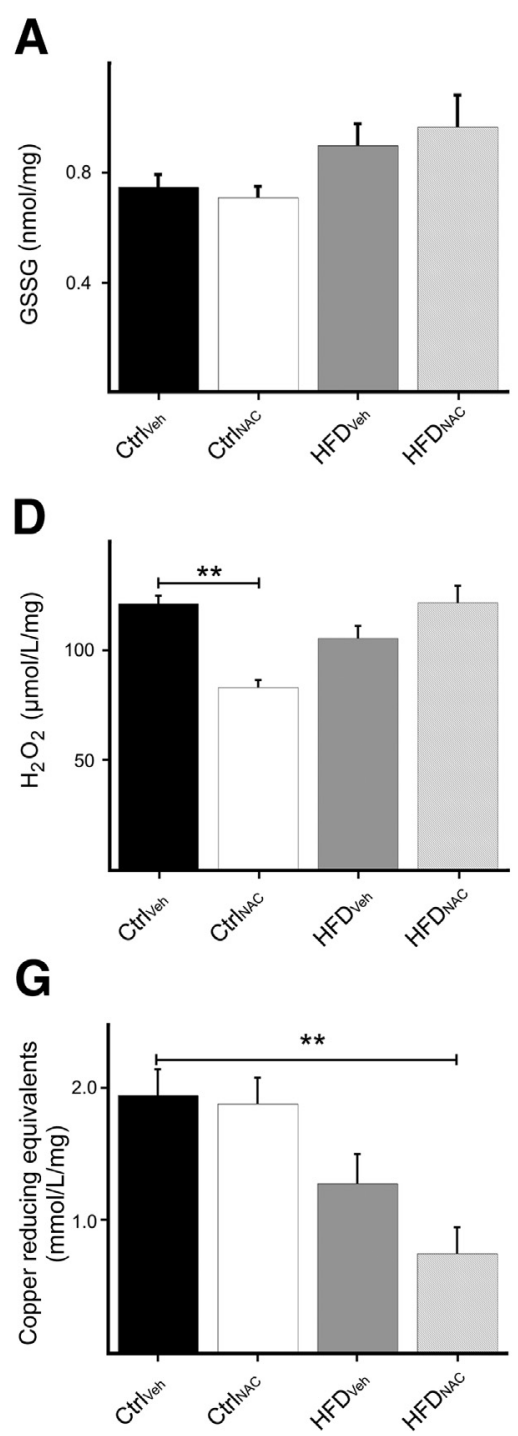
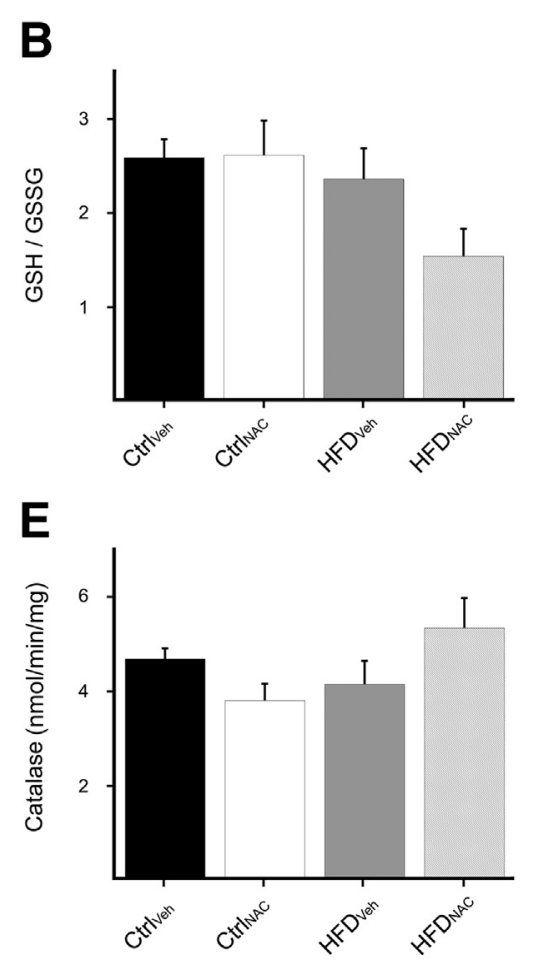
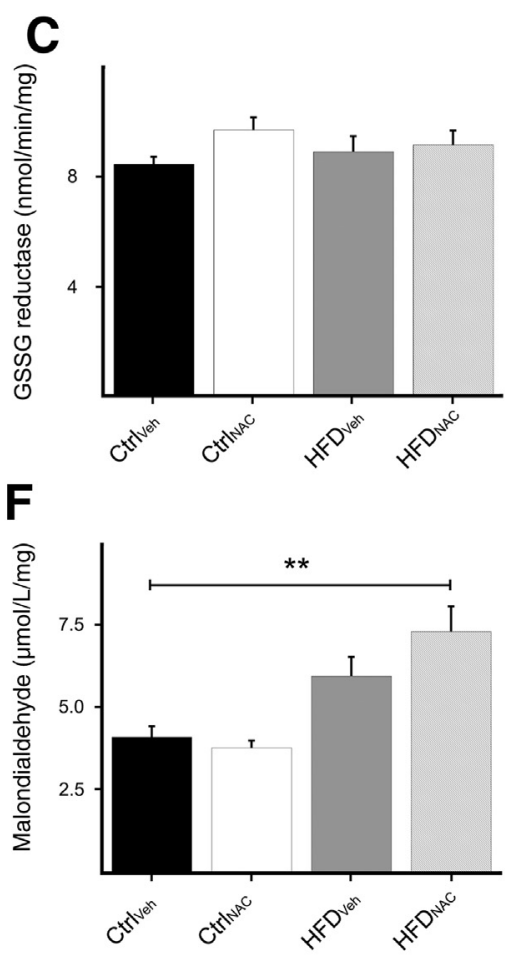

Figure 9 Interrogating oxidative stress markers in placental samples under control (Ctrl) and high-fat diet (HFD) conditions. A: Quantification of oxidized glutathione (GSSG) in placentas with or without $\mathrm{N}$-acetylcysteine (NAC) supplementation (NAC and Veh, respectively). B: Determination of the reduced glutathione (GSH)/GSSG ratio in placentas. C: Determination of GSSG reductase activity. D: Analysis of hydrogen peroxide levels within placentas. E: Probing catalase activity within placentas. F: Thiobarbituric acid reactive substances assay to evaluate levels of lipid peroxidation within placentas. G: Total antioxidant capacity measurements within placentas. ${ }^{* *} P<0.01$ (Tukey test).

occurring together in the maternal portion of the placenta and inflammatory cytokine expression in the fetal portion, both of which are ameliorated by NAC treatment. The addition of NAC prevented fibrinoid necrosis and partially resolved decidual inflammation in HFD dams. The compromised placental function in HFD dams likely plays a role in fetal growth restriction. Furthermore, although a difference in Ppar expression was not observed, Vegfa expression was significantly decreased in $\mathrm{HFD}_{\text {Veh }}$ placenta and normalized by NAC treatment. Vascular endothelial growth factor plays an important role in angiogenesis and is decreased in IUGR. ${ }^{34}$ Conditions associated with increased reactive oxygen species, such as preeclampsia and maternal obesity, are associated with alterations in Vegfa expression. $^{35}$ The physiologic role of placental growth factor, another member of the vascular endothelial growth factor family, is not well understood but is believed to control trophoblast growth and differentiation ${ }^{20,21}$ and stimulate angiogenesis. ${ }^{36}$ Although low placental growth factor expression is considered a marker of preeclampsia and deficient placental function, ${ }^{37} \mathrm{HFD}_{\text {veh }}$ resulted in increased P $f$ mRNA expression in our model. This finding may represent a compensatory mechanism because the ability to establish adequate fetoplacental vasculature is important for fetal development. The morphologic changes in the placental vasculature may result from dysregulation of vascular endothelial growth factor and likely lead to underperfusion of the placenta. In our model, HFD results in a significant increase in the frequency of inflammation and decidual vasculopathy occurring together, leading to defective nutrient transport and gas exchange, thus causing increased metabolic disease susceptibility in offspring.

Maternal proinflammatory cytokines can cross the placenta and impart inflammation on the fetus. In addition, 
they can cause indirect effects by altering placental nutrient flow. ${ }^{38-40}$ The extent of the effect of maternal inflammation on the fetus varies at different stages of pregnancy, depending on the organogenesis occurring at the time of insult. ${ }^{10}$ In agreement with the expected adverse effect of cytokines on placental perfusion, increased labyrinth immunostaining for IL-1 $\beta$ was observed in offspring exposed to $\mathrm{HFD}_{\mathrm{veh}}$, regardless of the sex or the genotype. Furthermore, maternal HFD resulted in increased expression of inflammatory signaling pathway genes $(\mathrm{Mcpl}, \mathrm{Il} 6, \mathrm{~N} f \kappa b$, Tlr4) and macrophage markers Adgrel and Itgax. NAC crosses the placenta and inhibits the placental inflammatory response. ${ }^{41}$ Interestingly, although maternal inflammatory cells were recruited to the decidua and expression of IL- $1 \beta$ was significantly increased in the labyrinth, no influx of immune cells to the labyrinth was observed. The most likely source of inflammatory cytokines in the labyrinth are trophoblasts because these cells are capable of immune responses. ${ }^{38,40}$ Surprisingly, placenta of $\mathrm{Ctrl}_{\mathrm{NAC}}$ dams exhibited increased expression of the proinflammatory cytokines Ill $\beta$, Tnf $\alpha$, and Itgax mRNAs.

Although the NAC treatment attenuated the increase in the HFD-induced increased frequency of inflammatorydecidual vasculopathy in WT dams, it did not prevent HFD-associated oxidative stress. In fact, NAC tended to intensify the prooxidant effects of the HFD. This paradoxical effect can be explained by NAC's capacity to autooxidize and convert from an antioxidant to a prooxidant in a very high-oxygen milieu. ${ }^{42}$ It is likely that the prooxidant effects of the maternal HFD, combined with the high levels of oxygen normally present in the maturing placenta, ${ }^{43}$ caused NAC to auto-oxidize. Remarkably, NAC was still able to partially reverse the placental morphologic changes provoked by maternal HFD, suggesting that this histopathologic finding can be at least partially resolved by suppressing the proinflammatory effects of HFD, even without fully reversing placental oxidative stress. Another factor that may have contributed to these paradoxical results is the fact that for the experiments depicted in Figure 9 whole placental explants were prepared. Decidua was not separated from the rest of the placenta. These two regions of the placenta may have significant differences in oxidative stress.

In summary, a maternal HFD in WT dams results in increased expression of placental inflammatory and oxidative stress genes, increased levels of labyrinthine IL-1 $\beta$, and an increase in the combination of inflammation and decidual vasculopathy, all resulting in reduced nutrient and oxygen delivery. The resolution of altered gene expression and the histopathologic features with NAC supports the hypothesis that inflammation and oxidative stress mediate the vascular changes that lead to the HFD-induced effects. To our knowledge, this is the first study to find that maternal HFD induces placental vasculopathy and treatment with NAC, despite its paradoxical prooxidant effects, has a profound ameliorative effect at both the molecular and morphologic levels. This study has several strengths: the use of an outbred mouse strain, blinding of the pathologist performing the histologic analysis, and investigation into amelioration of HFD-induced placental effects. This study focused on both histologic and molecular outcomes in placenta exposed to maternal HFD; however, the use of the entire placenta for gene expression assessments is a limitation of this study. Future studies focused on assessing localized regions within the placenta are warranted. Although it is established that exposure to an inflammatory environment during fetal development programs metabolic disease susceptibility, the mechanism by which the placenta responds to an altered IU milieu is complex and still poorly understood. Therefore, mechanistic studies such as this one, elucidating the pathway from exposure to an HFD to future development of metabolic disease, are necessary to develop new strategies to improve the IU environment and long-term health outcomes of offspring.

\section{Acknowledgment}

We thank Ernestine Middleton for preparing the microscopic slides.

L.W. performed most of the experiments and wrote the first draft of the manuscript; E.S.B. and C.R.M. performed the oxidative stress assays and some of the statistical analysis; R.P. assisted in the immunohistochemical experiments; P.V. assisted in obtaining the placentas and analyzed data; S.E.R. directed the histopathological analyses, performed the microscopy and scoring, edited the manuscript, and provided funding for histological processing; M.J.C. conceived the project, supervised all in vivo experiments, edited the manuscript, and provided most of the funding; M.J.C. is the guarantor of this work and, as such, had full access to all the data in the study and takes responsibility for the integrity of the data and the accuracy of the analysis.

\section{Supplemental Data}

Supplemental material for this article can be found at http://doi.org/10.1016/j.ajpath.2019.07.010.

\section{References}

1. Global Burden of Metabolic Risk Factors for Chronic Diseases Collaboration: Cardiovascular disease, chronic kidney disease, and diabetes mortality burden of cardiometabolic risk factors from 1980 to 2010: a comparative risk assessment. Lancet Diabetes Endocrinol 2014, 2:634-647

2. Aguilar M, Bhuket T, Torres S, Liu B, Wong RJ: Prevalence of the metabolic syndrome in the United States, 2003-2012. JAMA 2015, 313:1973-1974

3. Barker DJ, Gluckman PD, Godfrey KM, Harding JE, Owens JA, Robinson JS: Fetal nutrition and cardiovascular disease in adult life. Lancet 1993, 341:938-941

4. Barker DJ, Martyn CN: The maternal and fetal origins of cardiovascular disease. J Epidemiol Community Health 1992, 46:8-11 
5. Block T, El-Osta A: Epigenetic programming, early life nutrition and the risk of metabolic disease. Atherosclerosis 2017, 266:31-40

6. Calkins K, Devaskar SU: Fetal origins of adult disease. Curr Probl Pediatr Adolesc Health Care 2011, 41:158-176

7. El Hajj N, Schneider E, Lehnen H, Haaf T: Epigenetics and life-long consequences of an adverse nutritional and diabetic intrauterine environment. Reproduction 2014, 148:R111-R120

8. Hoy WE, Nicol JL: The Barker hypothesis confirmed: association of low birth weight with all-cause natural deaths in young adult life in a remote Australian Aboriginal community. J Dev Origins Health Dis $2019,10: 55-62$

9. Hartil K, Vuguin PM, Kruse M, Schmuel E, Fiallo A, Vargas C, Warner MJ, Durand JL, Jelicks LA, Charron MJ: Maternal substrate utilization programs the development of the metabolic syndrome in male mice exposed to high fat in utero. Pediatr Res 2009, 66:368-373

10. Plata M, Williams L, Seki Y, Hartil K, Kaur H, Lin CL, Fiallo A, Glenn AS, Katz EB, Fuloria M, Charron MJ, Vuguin PM: Critical periods of increased fetal vulnerability to a maternal high fat diet. Reprod Biol Endocrinol 2014, 12:80

11. Vuguin PM, Hartil K, Kruse M, Kaur H, Lin CL, Fiallo A, Glenn AS, Patel A, Williams L, Seki Y, Katz EB, Charron MJ: Shared effects of genetic and intrauterine and perinatal environment on the development of metabolic syndrome. PLoS One 2013, 8:e63021

12. Dimasuay KG, Boeuf P, Powell TL, Jansson T: Placental responses to changes in the maternal environment determine fetal growth. Front Physiol 2016, 7:12

13. Burton GJ, Jauniaux E: Oxidative stress. Best Pract Res Clin Obstet Gynaecol 2011, 25:287-299

14. Deepmala, Slattery J, Kumar N, Delhey L, Berk M, Dean O, Spielholz C, Frye R: Clinical trials of N-acetylcysteine in psychiatry and neurology: a systematic review. Neurosci Biobehav Rev 2015, $55: 294-321$

15. Paintlia MK, Paintlia AS, Singh AK, Singh I: Attenuation of lipopolysaccharide-induced inflammatory response and phospholipids metabolism at the feto-maternal interface by $\mathrm{N}$-acetyl cysteine. Pediatr Res 2008, 64:334-339

16. Beloosesky R, Gayle DA, Amidi F, Nunez SE, Babu J, Desai M, Ross MG: N-acetyl-cysteine suppresses amniotic fluid and placenta inflammatory cytokine responses to lipopolysaccharide in rats. Am J Obstet Gynecol 2006, 194:268-273

17. Amin AF, Shaaban OM, Bediawy MA: N-acetyl cysteine for treatment of recurrent unexplained pregnancy loss. Reprod Biomed Online 2008, 17:722-726

18. Groom KM, David AL: The role of aspirin, heparin, and other interventions in the prevention and treatment of fetal growth restriction. Am J Obstet Gynecol 2018, 218:S829-S840

19. Committee for the Update of the Guide for the Care and Use of Laboratory Animals; National Research Council: Guide for the Care and Use of Laboratory Animals: Eighth Edition. Washington, DC, National Academies Press, 2011

20. De Falco $S$ : The discovery of placenta growth factor and its biological activity. Exp Mol Med 2012, 44:1-9

21. Khaliq A, Li XF, Shams M, Sisi P, Acevedo CA, Whittle MJ, Weich H, Ahmed A: Localisation of placenta growth factor (PIGF) in human term placenta. Growth Factors 1996, 13:243-250

22. Williams L, Charron MJ, Sellers RS: High postnatal mortality associated with defects in lung maturation and reduced adiposity in mice with gestational exposure to high fat diet and NAC. Res Vet Sci 2017, 114:262-265

23. McCurdy CE, Bishop JM, Williams SM, Grayson BE, Smith MS, Friedman JE, Grove KL: Maternal high-fat diet triggers lipotoxicity in the fetal livers of nonhuman primates. J Clin Invest 2009, 119:323-335

24. Frias AE, Morgan TK, Evans AE, Rasanen J, Oh KY, Thornburg KL, Grove KL: Maternal high-fat diet disturbs uteroplacental hemodynamics and increases the frequency of stillbirth in a nonhuman primate model of excess nutrition. Endocrinology 2011, 152: $2456-2464$

25. Grayson BE, Levasseur PR, Williams SM, Smith MS, Marks DL, Grove KL: Changes in melanocortin expression and inflammatory pathways in fetal offspring of nonhuman primates fed a high-fat diet. Endocrinology 2010, 151:1622-1632

26. Sullivan EL, Grayson B, Takahashi D, Robertson N, Maier A, Bethea CL, Smith MS, Coleman K, Grove KL: Chronic consumption of a high-fat diet during pregnancy causes perturbations in the serotonergic system and increased anxiety-like behavior in nonhuman primate offspring. J Neurosci 2010, 30:3826-3830

27. Giriko CA, Andreoli CA, Mennitti LV, Hosoume LF, Souto Tdos S, Silva AV, Mendes-da-Silva C: Delayed physical and neurobehavioral development and increased aggressive and depression-like behaviors in the rat offspring of dams fed a high-fat diet. Int J Dev Neurosci 2013, 31:731-739

28. Sun B, Purcell RH, Terrillion CE, Yan J, Moran TH, Tamashiro KL: Maternal high-fat diet during gestation or suckling differentially affects offspring leptin sensitivity and obesity. Diabetes 2012, 61: 2833-2841

29. Roos S, Powell TL, Jansson T: Placental mTOR links maternal nutrient availability to fetal growth. Biochem Soc Trans 2009, 37: 295-298

30. Eriksson JG, Kajantie E, Thornburg KL, Osmond C, Barker DJ: Mother's body size and placental size predict coronary heart disease in men. Eur Heart J 2011, 32:2297-2303

31. Heshmati A, Koupil I: Placental weight and foetal growth rate as predictors of ischaemic heart disease in a Swedish cohort. J Dev Origins Health Dis 2014, 5:164-170

32. Mark PJ, Sisala C, Connor K, Patel R, Lewis JL, Vickers MH, Waddell BJ, Sloboda DM: A maternal high-fat diet in pregnancy reduces growth of the fetus and the placental junctional zone, but not placental labyrinth zone growth. J Dev Origins Health Dis 2011, 2:63-70

33. Schulz LC, Schlitt JM, Caesar G, Pennington KA: Leptin and the placental response to maternal food restriction during early pregnancy in mice. Biol Reprod 2012, 87:120

34. Hewitt DP, Mark PJ, Waddell BJ: Glucocorticoids prevent the normal increase in placental vascular endothelial growth factor expression and placental vascularity during late pregnancy in the rat. Endocrinology 2006, 147:5568-5574

35. Pereira RD, De Long NE, Wang RC, Yazdi FT, Holloway AC, Raha S: Angiogenesis in the placenta: the role of reactive oxygen species signaling. Biomed Res Int 2015, 2015:814543

36. Maynard SE, Karumanchi SA: Angiogenic factors and preeclampsia. Semin Nephrol 2011, 31:33-46

37. Welch PC, Amankwah KS, Miller P, McAsey ME, Torry DS: Correlations of placental perfusion and PlGF protein expression in early human pregnancy. Am J Obstet Gynecol 2006, 194:1625-1629. discussion 1629-1631

38. Guleria I, Pollard JW: The trophoblast is a component of the innate immune system during pregnancy. Nat Med 2000, 6:589-593

39. Ingvorsen C, Brix S, Ozanne SE, Hellgren LI: The effect of maternal inflammation on foetal programming of metabolic disease. Acta Physiol 2015, 214:440-449

40. Mor G, Cardenas I: The immune system in pregnancy: a unique complexity. Am J Reprod Immunol 2010, 63:425-433

41. Horowitz RS, Dart RC, Jarvie DR, Bearer CF, Gupta U: Placental transfer of N-acetylcysteine following human maternal acetaminophen toxicity. J Toxicol Clin Toxicol 1997, 35:447-451

42. Chan ED, Riches DW, White CW: Redox paradox: effect of $\mathrm{N}$-acetylcysteine and serum on oxidation reduction-sensitive mitogen-activated protein kinase signaling pathways. Am J Respir Cell Mol Biol 2001, 24:627-632

43. Wu F, Tian FJ, Lin Y: Oxidative stress in placenta: health and diseases. Biomed Res Int 2015, 2015:293271 